\title{
Malignant Shoulder Syndrome: An Under-Appreciated Feature of Renal Cell Carcinoma
}

\author{
Michael Jerome McKaya, ${ }^{\mathrm{a}}$, Kirsty Rady ${ }^{\mathrm{b}}$
}

\begin{abstract}
We coin the term Malignant Shoulder Syndrome to describe a predilection of renal cell cancer for metastasizing to the shoulder girdle, and in particular the scapula. Here we describe a patient with the novel feature of bilateral shoulder involvement from metastatic renal cell cancer. Hypotheses for this phenomenon are presented.
\end{abstract}

Keywords: Renal cell cancer; Metastasis; Malignant shoulder syndrome

\section{Introduction}

Skeletal metastases are a common feature of renal cell carcinoma (RCC) $[1,2]$. In particular, the majority of scapular metastases from RCC are large and highly symptomatic. We previously noted such an unusual propensity for RCC to metastasise to the scapula [3] and describe another (novel, bilateral) case here.

A 76-year-old man admitted to hospital who had has no previous malignancy presented with clinical and radiographic evidence of probable metastatic destruction of much of the sacrum. Core needle biopsy was non-diagnostic. On $\mathrm{CT}$, a heterogeneous low density mass within the right kidney suggested RCC, biopsy of which demonstrated clear cell carcinoma. Radiotherapy was delivered to the sacrum but the patient refused systemic therapy.

\footnotetext{
Manuscript accepted for publication August 3, 2013

${ }^{a}$ North Coast Cancer Institute, Lismore 2480, New South Wales, Australia

${ }^{\mathrm{b}}$ Department of Radiation Oncology, Canberra Hospital, Garran 2605, Australian Capital Territory, Australia

${ }^{\mathrm{c} C}$ Corresponding author: Michael Jerome McKay, North Coast Cancer Institute, 70-72 Hunter St, Lismore 2480, New South Wales, Australia. Email: cohesin@yahoo.com.au
}

doi: http://dx.doi.org/10.4021/jmc1467w
He developed right shoulder pain, plain radiographs showing a destructive bone lesion involving the right scapular spine, confirmed on radiotherapy simulation films (Fig. 1A). The patient then suffered pathological fractures both to the acromion of the right scapula and the right humeral head. The latter was internally fixated. He received palliative radiotherapy to these sites. Four months later he developed severe pain in his contralateral shoulder. X-rays and subsequent radiotherapy simulation films showed significant destruction of most of the main bony components of his left shoulder, including humeral head and lateral clavicle, with a major destructive lesion in the spine of the left scapula (Fig. 1B). The patient's condition deteriorated and he died some weeks later.

\section{Discussion}

This case emphasises an uncommonly recognised but clinically important pattern of metastatic spread of RCC: to the shoulder girdle and in particular, the scapula $[2,3]$. We propose the name 'malignant shoulder syndrome' (MSS) for this entity. RCC typically metastasises, via both lymphatic and venous routes to lung parenchyma, liver, brain, and bone. Around one-third of patients with RCC develop skeletal metastases, almost one-half suffer pathological long-bone fracture and around one-third require orthopaedic intervention $[2,3]$. Bone pain and spinal cord compression are significant causes of morbidity in these patients. Metastatic RCC to the shoulder can also be a presenting feature [4], illustrating that recognition of MSS is important, especially in the context of a characteristic associated vascular or pulsatile RCC metastatic mass.

The reason for the predilection of metastatic RCC spread to the shoulder region remains unknown. Anastamoses between the venous drainage of the kidney and the ipsilateral scapula is a proposed contributor [2, 3], consistent with the 'preferential circulation' hypothesis of Virchow (see http//:en/wikipedia.org/wiki/Rudolph_Virchow). However, the lack of co-lateralisation with primary tumours $[2,3]$ would argue against this as a sole mechanism. Another potential mechanism could relate to the 'seed and soil hypoth- 

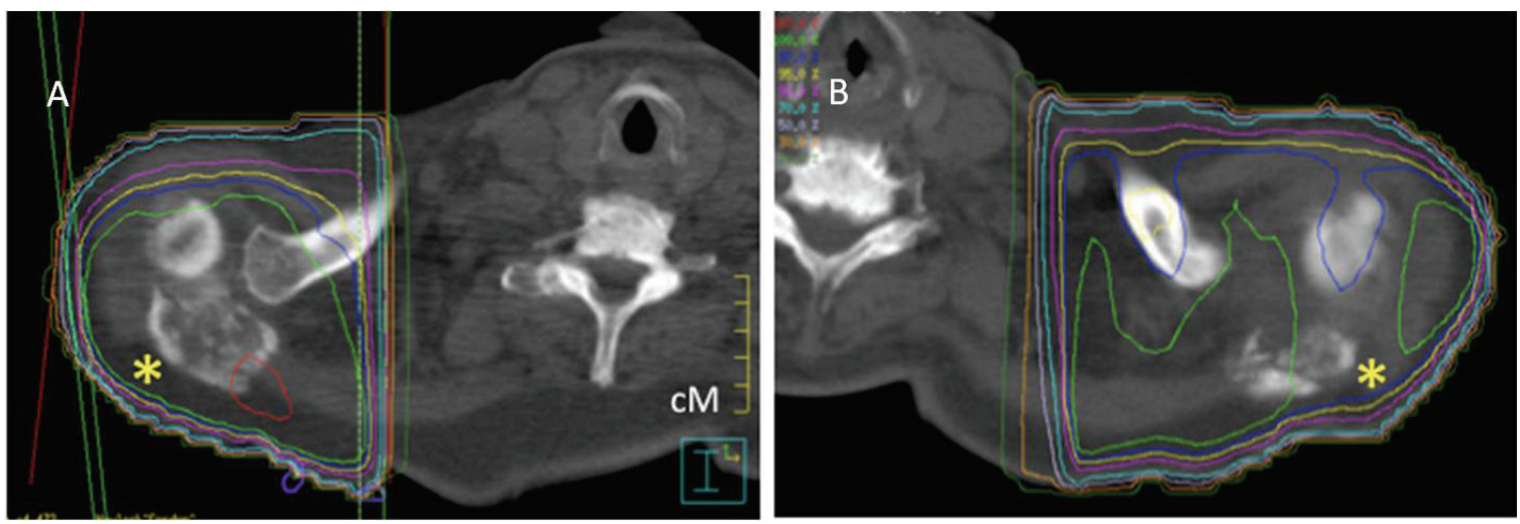

Figure 1. Axial CT of shoulder regions showing bilateral (A: right and B: left) comminuting infiltration of the lateral scapular spines by metastatic RCC (asterisks). Some adjacent shoulder bones are also affected. Coloured lines represent radiotherapy dose distribution.

esis' where tumour cells are postulated to have an affinity for the milieu of specific organs, first suggested by the English surgeon, Stephen Paget [5]. It remains unknown which, if either, proposed mechanism might explain MCC, the predilection of RCC for shoulder region metastases.

\section{References}

1. Breau RH, Blute ML. Surgery for renal cell carcinoma metastases. Curr Opin Urol. 2010;20(5):375-381.

2. Jacobsen KD, Folleras G, Fossa SD. Metastases from renal cell carcinoma to the humerus or the shoulder girdle.
Br J Urol. 1994;73(2):124-128.

3. Gurney H, Larcos G, McKay M, Kefford R, Langlands A. Bone metastases in hypernephroma. Frequency of scapular involvement. Cancer. 1989;64(7):1429-1431.

4. Placed IG, Alvarez-Rodriguez R, Pombo-Otero J, Vazquez-Bartolome P, Hermida-Romero T, PomboFelipe F. Metastatic renal cell carcinoma presenting as shoulder monoarthritis: diagnosis based on synovial fluid cytology and immunocytochemistry. Acta Cytol. 2010;54(5):730-733.

5. Paget $\mathrm{S}$. The distribution of secondary growths in cancer of the breast. 1889. Cancer Metastasis Rev. 1989;8(2):98-101. 